RESEARCH PAPER

J. Food Sci. Technol. Nepal, Vol. 8 (23-29 ) 2013

ISSN: 1816-0727

\title{
Characterization and Comparison of Soy Milk and Buffalo Milk Based Synbiotic Product
}

\author{
TIKA KARKI*, SHILVA SHRESTHA, BINITA BOHARA and UJWAL JYAKHWA \\ Department of Biotechnology, School of Science, Kathmandu University, Dhulikhel, Nepal
}

*Corresponding author: ktika@ku.edu.np

Today consumers prefer foods that not only provide basic nutrition but also contain compounds that confer health benefits. Among the functional food, synbiotic products are showing a promising future. Soy based synbiotic products using different probiotics: A1B and MgA (lactobacillus strains) and L.rhamanosus ATCC 9595 and prebiotics: lactulose, sunfibre and inulin were prepared and its antiradical activities along with other characteristics were studied. The study also aimed to determine the most suitable prebiotics and probiotics. Furthermore, the prebiotics and the organisms were also cultured in buffalo milk to compare its properties with synbiotic soy yoghurt. Synbiotic soy yoghurt showed highest anti-radical activity with \% inhibition at the range of 41-45\% and the one with probiotic L.rhamnosus and prebiotic inulin was found to be the most suitable product.

Keywords: Synbiotic, Probiotic, Prebiotic, Inulin, Anti-radical

\section{Introduction}

Soy milk is very nutritive: it's a good source of high quality proteins, isoflavones and B-vitamins. It is free of milk sugar (lactose) and is a good choice for people who are lactose intolerant. Consumers in western countries consume soymilk mainly as an important replacer of cow milk due to lactose intolerance or allergic reaction to cow's milk, and as a low cost source of good quality protein and energy (Rosenthal et al., 2003; Lui, 1997; Kwok and Niranjan, 1995; Kanthamani et al., 1978). Soy milk has antiradical activity, through the isoflavones, which acts as an effective reducing tool for oxidative degradation of DNA, prevention of premature aging and the emergence of diseases like Alzheimer's (Hsieh et al., 2009). Fermented soymilk have numerous advantages over nonfermented one (Chow, 2002). Fermentation may reduce flatulence, destroy undesirable pathogens, improve product flavor and reduce beany flavor, give new textures, and, when un-pasteurized, protect those who have eaten it from intestinal infections, and help replenish the intestinal flora (Trindade et al., 2001). Lactic acid fermentation has been reported as a means to reduce beany flavors. Consequently, soy milk based yoghurts offer a considerable appeal for a growing segment of consumers with certain dietary and health concern. In addition, it has several nutritional advantages over cow milk yoghurt such as, reduced levels of cholesterol, of saturated fat and free of lactose (Pyo and Song, 2009).
A synbiotic is a supplement that contains both a prebiotic and a probiotic that work together to improve the "friendly flora" of the human intestine. The main reason for using a synbiotic is that a true probiotic, without its prebiotic food, does not survive well in the digestive system. To enhance viability, not only on the shelf but also in the colon, the product must allow for much greater attachment and growth rate of the healthy bacteria in order to minimize the growth of harmful bacteria. Without the necessary food source for the probiotic, it will have a greater intolerance for oxygen, low $\mathrm{pH}$, and temperature. In addition, the probiotic will have to compete against other bacteria that will take over if its specific food source is not available (Verma and Palanchoke, 2007) . Therefore, a "synbiotic" product (probiotic + prebiotic $=$ synbiotic) makes for a better choice.

The objective of this study was to develop soy based synbiotic yoghurt with good acceptability using different probiotics and prebiotics, to determine the most suitable probiotic and prebiotic as well as compare the synbiotic soy yoghurt with buffalo milk based synbiotic yoghurt and finally evaluate its different properties specifically anti-radical activity.

\section{Materials and Methods}

\section{Prebiotics (Microorganisms) and Probiotics}

Lactobacillus rhamnosus ATCC 9595, A1B and MgA (lactobacillus strains) were used for experiment. A1B and $\mathrm{MgA}$ strains cultures were obtained from National 
Karki et al.: J. Food Sci. Technol. Nepal, Vol. 8 (23-29 ), 2013

Institute of Science and Technology (NIST), Nepal. All three strains were sub cultured in MRS agar media containing $1 \%$ calcium carbonate. Inulin, Lactulose and Sunfibre were used as prebiotics.

\section{Preparation of soymilk}

Soy milk was prepared in the laboratory using white soybean variety. Soybean soaked in water for $16 \mathrm{hr}$ was decapitated and boiled in $1 \%$ sodium bicarbonate for $5 \mathrm{~min}$ and subsequently washed with water. It was then grinded along with warm water ( $7: 1$ ratio) and the resulting soymilk was filtered through cheese cloth. It was finally sterilized by autoclaving and stored in refrigerator at $4^{\circ} \mathrm{C}$.

Soy milk/buffalo milk based synbiotic and probiotic products preparation

Soymilk/Buffalo milk was heated at $110-112^{\circ} \mathrm{C}$ and poured into culture tubes into which $1 \%$ prebiotics (inulin, lactulose and sunfibre) was added. The strains (L.rhamnosus, $\mathrm{A} 1 \mathrm{~B}$ and $\mathrm{MgA}$ )were then inoculated with $6 \log \mathrm{CFU} / \mathrm{ml}$ into it, stirred thoroughly and incubated at $37^{\circ} \mathrm{C}$ for $24 \mathrm{hr}$ to obtain synbiotic and probiotic yoghurts. Furthermore, different properties of thus prepared synbiotic soymilk and buffalo milk were studied and compared with each other as well as with Juju dhau (king curd from Bhaktapur) and probiotic curd from DDC.

Twenty four different synbiotic and probiotic soy yoghurt and buffalo yoghurt prepared in our laboratory are listed below.

A. Lactobacillus rhamnosus with $1 \%$ inulin for both soy milk and buffalo milk

B. Lactobacillus rhamnosus with $1 \%$ lactulose for both soy milk and buffalo milk

C. Lactobacillus rhamnosus with $1 \%$ sunfibre for both soy milk and buffalo milk

D. Lactobacillus rhamnosus for probiotic soy milk and probiotic buffalo milk

E. A1B strain with $1 \%$ inulin for both soy milk and buffalo milk

F. A1B strain with $1 \%$ lactulose for both soy milk and buffalo milk

G. A1B strain with $1 \%$ sunfibre for both soy milk and buffalo milk

H. A1B strain for probiotic soy milk and probiotic buffalo milk

I. MgA strain with $1 \%$ inulin for both soy milk and buffalo milk
J. MgA strain with $1 \%$ lactulose for both soy milk and buffalo milk

K. MgA strain with $1 \%$ sunfibre for both soy milk and buffalo milk

L. MgA strain for probiotic soy milk and probiotic buffalo milk

\section{Biochemical changes during fermentation of Synbiotic and Probiotic Products \\ pH and Titratable acidity}

$\mathrm{pH}$ measurements were carried out at room temperature $\left(27^{\circ} \mathrm{C}\right)$ by means of a digital $\mathrm{pH}$ meter(HANNA instruments $\mathrm{pH} 211$ Microprocessor $\mathrm{pH}$ meter) (AOAC, 2005), 24 hours from incubation, after 5 days and after 10 days from incubation.

Titratable acidity was measured by titration with $0.1 \mathrm{~N}$ sodium hydroxide solutions and using $1 \%$ ethanol solution of phenolphthalein as indicator (Agrarwala and Sharma, 1961). It was also measured 24 hours from incubation, after 5 days and after 10 days from incubation.

\section{Total soluble solids (TSS)}

TSS was determined by using refractometer (portable refractometer, model: FG 103, Brix 0-32\%; Comecta S. A.) (Daniel 2010) in terms of degrees Brix for all soy and buffalo yoghurt samples along with Juju dhau (king curd) and prebiotic yoghurt from DDC.

Viability of Probiotic organisms present in synbiotic and probiotic yoghurts

MRS agar containing 1\% calcium carbonate was used for propagation of the lactobacilli strains in the samples. Standard Plate Count (SPC) method (Eaton et al., 1960) was used. After incubation, the inoculated plates having 30 to 300 colonies were considered for counting and expressed as $\log \mathrm{CFU} / \mathrm{ml}$ of soymilk.

\section{Anti-radical activity}

1, 1-Diphenyl-2-picryl-hydrazyl (DPPH) is free radical but stable. DPPH solution is initially violet in color which fades when antioxidants donate hydrogen. The change in color is monitored by spectrophotometer. DPPH free radical scavenging activity was calculated on the basis of the method described by Molyneux (2004).

\section{Result and Discussion \\ Change in $\mathrm{pH}$}

The change in $\mathrm{pH}$ was determined after 1 day, 5 days and the 10 days of fermentation. The $\mathrm{pH}$ showed decreasing trend with the progression of fermentation (Fig 1, Fig2 and Fig 3). Synbiotic soy yoghurt with lactulose as 
prebiotic and L.rhamnosus as probiotic showed decreases in $\mathrm{pH}$ (4.94 to 4.30) slightly more than other synbiotic soy yoghurts. Similarly pH of synbiotic buffalo yoghurt with prebiotic lactulose and probiotic L.rhamnosus decreased (4.51 to 4.12) slightly more than other products. The $\mathrm{pH}$ of probiotic soy yoghurt and probiotic buffalo yoghurt both with L.rhamnosus showed decrease in $\mathrm{pH}$ from 5.20 to 4.85 and 4.82 to 4.57 respectively which was slightly more than other similar probiotic soy yoghurt and buffalo yoghurt. The $\mathrm{pH}$ of Juju dhau decreased from 4.50 to 4.21 and that of probiotic yoghurt (DDC) from 4.49 to 4.25. The $\mathrm{pH}$ of the product with L.rhamnosus, $A 1 B$ strain and $\mathrm{MgA}$ was almost similar for prebiotic lactulose but different from sunfibre and inulin. Inulin containing yoghurt was found to exhibit low $\mathrm{pH}$ when compared with samples containing lactulose and sunfibre. Production of lactic acid in the samples was found to be influenced by the prebiotic used. The lowest difference in $\mathrm{pH}$ was found around 0.24 in probiotic yoghurt (DDC) which resembles very close to probiotic buffalo yoghurt with L rhamnosus.

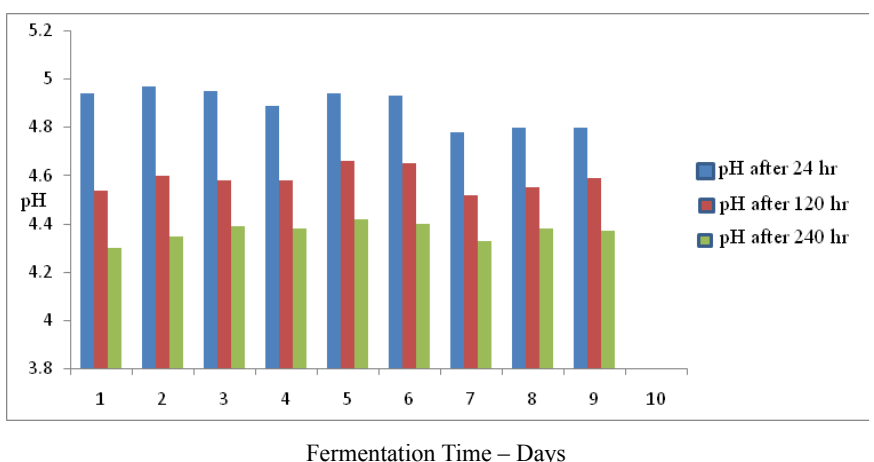

Figure 1. pH of Soymilk based Synbiotic Product

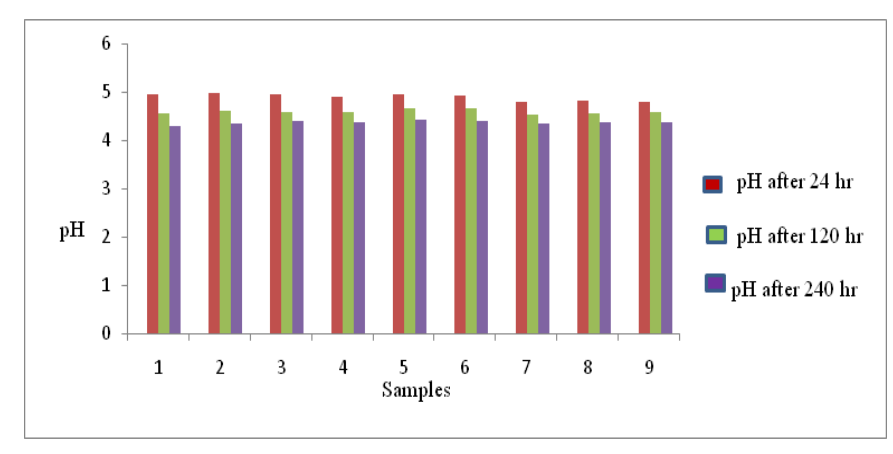

Figure 2. pH of Buffalo Milk based Synbiotic Product

For Figure 1 and 2:

1: Lactulose + L.rhamnosus 6: Sun Fibre + MgA strain

2: Lactulose + A1B strain 7: Inulin + L.rhamnosus

3: Lactulose $+\mathrm{MgA}$ strain 8: Inulin $+\mathrm{A} 1 \mathrm{~B}$ strain

4: Sun Fibre + L.rhamnosus 9: Inulin $+\mathrm{MgA}$ strain

5: Sun Fibre + A1B strain

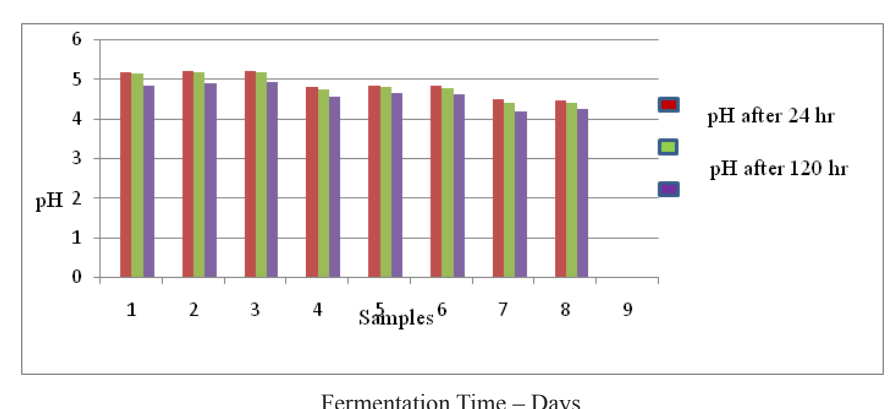

Figure 3. pH of Soymilk and Buffalo Milk based Probiotic product, King Curd and Probiotic Yoghurt (DDC)

1: Soymilk + L.rhamnosus 5: Buffalo milk + A1B strain 2: Soymilk + A1B strain 6: Buffalo milk $+\mathrm{MgA}$ strain 3: Soymilk + MgA strain 7: King Curd 4: Buffalo milk + L.rhamnosus $\quad$ 8: Probiotic Yoghurt (DDC)

\section{Change in Titratable Acidity}

The titratable acidity of soy based synbiotic yoghurt with L.rhamnosus as probiotic and inulin as prebiotic showed highest acidity with 0.315 to $0.360 \%$ and the one with $\mathrm{MgA}$ strain as probiotic and lactulose as prebiotic showed lowest acidity with 0.279 to $0.321 \%$ (Fig 4). Similarly, Buffalo based synbiotic yoghurt with L.rhamnosus as probiotic and lactulose as prebiotic showed highest production of lactic acid with change in titratable acidity from $0.315 \%$ to $0.380 \%$ after 10 days while the one with prebiotic sunfibre and probiotic $\mathrm{MgA}$ strain showed lowest value with increase in titratable acidity from 0.288 $\%$ to $0.335 \%$ (Fig 5). For both probiotic soy yoghurt and probiotic buffalo yoghurt product with L.rhamnosus showed highest acid production with change in titratable acidity from $0.270 \%$ to $0.327 \%$ and $0.297 \%$ to $0.332 \%$ respectively among the different probiotic yoghurts (Fig 6). Juju dhau showed acidity of 0.360 to $0.416 \%$ and probiotic yoghurt (DDC) had acidity of 0.342 to $0.408 \%$.

Result showed that L.rhamnosus produced more lactic acid than $\mathrm{MgA}$ and $\mathrm{A} 1 \mathrm{~B}$ strain either by addition of prebiotics or without addition of prebiotics. Titratable acidity depends on the amount of carbohydrate contained in the yoghurt samples, lactose content in buffalo milk is high thus buffalo based yoghurts showed high titratable acidity compare to soy yoghurts. 


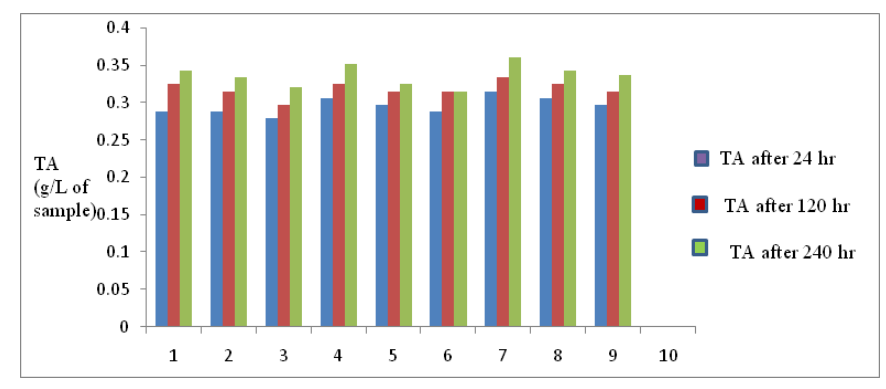

Fermentation Time - Days

Figure 4. Titratable acidity (TA) of Soymilk based Synbiotic Product

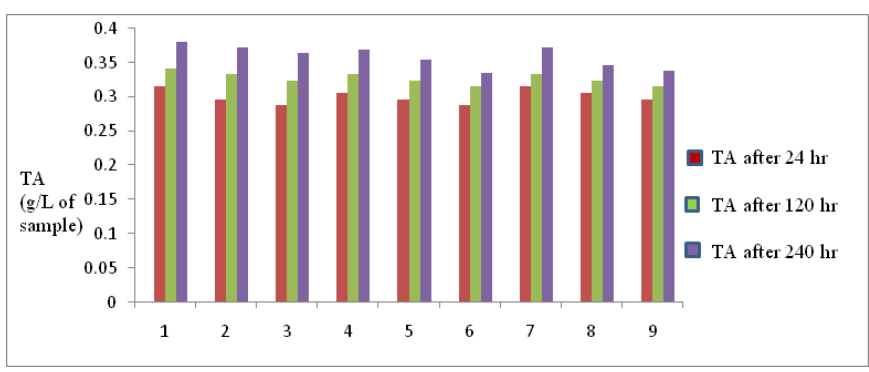

Fermentation Time - Days

Figure 5. Titratable acidity (TA) of Buffalo Milk based Synbiotic Product

For Fig 4 and 5:

1: Lactulose + L.rhamnosus 6: Sun Fibre + MgA strain

2: Lactulose + A1B strain 7: Inulin + L.rhamnosus

3: Lactulose + MgA strain 8: Inulin + A1B strain

4: Sun Fibre + L.rhamnosus 9: Inulin $+\mathrm{MgA}$ strain

5: Sun Fibre + A1B strain

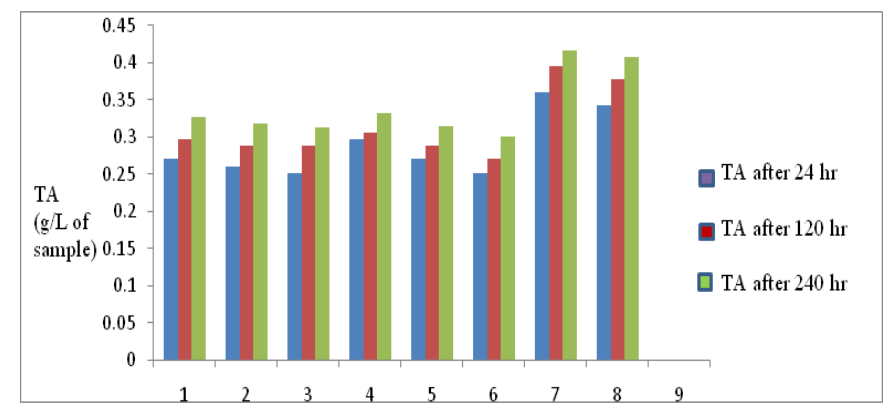

Figure 6. Titratable acidity (TA) of Soymilk, Buffalo Milk based probiotic product, King Curd and Probiotic yoghurt (DDC)

1: Soymilk + L.rhamnosus 5: Buffalo milk + A1B strain

2: Soymilk + A1B strain 6: Buffalo milk $+\mathrm{MgA}$ strain

3: Soymilk + MgA strain 7: King Curd

4: Buffalo milk + L.rhamnosus 8: Probiotic Yoghurt (DDC)

\section{Total Soluble Solid}

Figure 7 Shows that Buffalo milk based synbiotic product containing lactulose as prebiotic and A1B strain as probiotic and the one with sunfibre as prebiotic and L.rhamnosus as probiotic had the highest solid content around $11.4 \%$ However, the Synbiotic soy yoghurt containing lactulose as prebiotic and L.rhamnosus as probiotic, sunfibre as prebiotic and $\mathrm{MgA}$ as probiotic and inulin as prebiotic and L.rhamnosus as probiotic was found to contain solid content around $6.2 \%$. Similarly, probiotic soy yoghurt with L.rhamnosus as the probiotic showed the lowest solid content of $2.4 \%$.among all other probiotic soy products. Similarly, total solid content of probiotic buffalo yoghurt containing L.rhamnosus was found highest with value 8.2.The total soluble solid content of Juju dhau and Probiotic yoghurt from DDC was found to be 15.4 and 9.6 respectively.

From the observations of all the yoghurt samples, it was found that Juju dhau had the highest total solid content; which might be due to the addition of sugar at higher concentration ( $1 \mathrm{~kg}$ sugar in 20 litres of milk) compared to other yoghurts in which no sugar was added. Also, buffalo yoghurt had higher solid content than the soy yoghurt which may be due to presence of lactose in buffalo milk and other carbohydrates which is absent or present in considerable fewer amounts in soy milk.

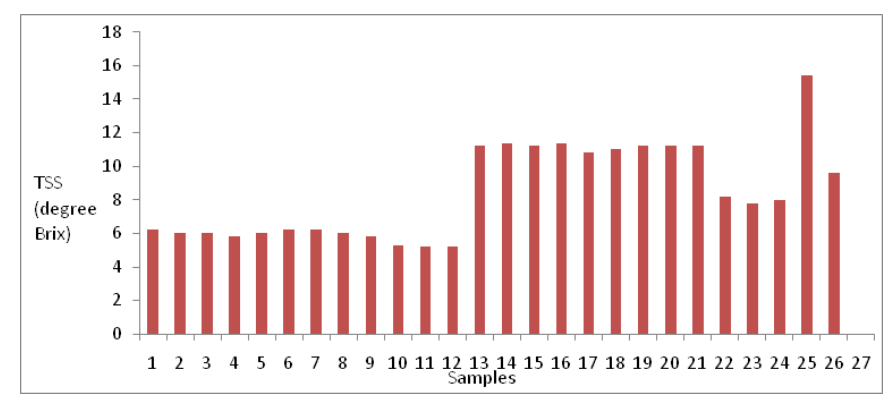

Figure 7. Total Soluble Solid ( ${ }^{0}$ Brix) of different products

Soy Milk: 1: Lactulose + L.rhamnosus, 2: Lactulose + A1B strain, 3: Lactulose + MgA strain, 4: Sun Fibre + L.rhamnosus, 5: Sun Fibre + A1B strain, 6: Sun Fibre + MgA strain, 7: Inulin + L.rhamnosus, S8: Inulin + A1B strain, 9: Inulin + MgA strain

Buffalo Milk: 10: Lactulose + L.rhamnosus, 11: Lactulose + A1B strain, 12: Lactulose + MgA strain, 13: Sun Fibre + L.rhamnosus, 14: Sun Fibre + A1B strain, 15: Sun Fibre + MgA strain, 16: Inulin + L.rhamnosus, 17: Inulin + A1B strain, 18: Inulin + MgA strain

Probiotics: 19: Soymilk + L.rhamnosus, 20: Soymilk + A1B strain, 21: Soymilk + MgA strain 22: Buffalo milk + L.rhamnosus, 23: Buffalo milk + A1B strain, 24: Buffalo milk + MgA strain, 25: King Curd, 26: Probiotic Yoghurt (DDC) 
Karki et al.: J. Food Sci. Technol. Nepal, Vol. 8 (23-29 ), 2013

\section{Viability of Probiotic Organisms present in different products}

The viability of different probiotic strains present in all the samples, after 1 day, 5 days, 10 days and 15 days of fermentation was deteermined. Clear zones of colonies were observed in MRS media. According to our observation (Fig 8), probiotic strain present in both buffalo based and soy based synbiotic product maintained $8 \operatorname{logs} \mathrm{cfu} / \mathrm{ml}$ till 15 days from incubation. Similar result was observed in case of Juju dhau ( 8 logs CFU/ml for 15 days). The stability of probiotics present in the probiotic yoghurt from DDC decreased with the number of days i.e. by a log cycle from 8.46 logs CFU/ml to 7.27 logs CFU/ml in 10 days, showing viability till $5^{\text {th }}$ day from incubation. This decrease may be due to the low survivability rate of the probiotics used in the yoghurt. The viability of the probiotic soy yoghurt and the probiotic buffalo yoghurt was reduced after $10^{\text {th }}$ day where as the viability of the synbiotic soy yoghurt, synbiotic buffalo yoghurt and Juju Dhau were seen to be stable till the $10^{\text {th }}$ and $15^{\text {th }}$ day.

So, it can be inferred that the presence of prebiotics in synbiotic yoghurt enhanced the viability of the probiotic strain present in it because of their ability to be fermented by the lactobacilli strains. In the presence of inulin, sunfibre and lactulose, cultures showed better retention of viability $(8.0 \log \mathrm{cfu} / \mathrm{ml})$ in comparison to that of the yoghurts without prebiotics.

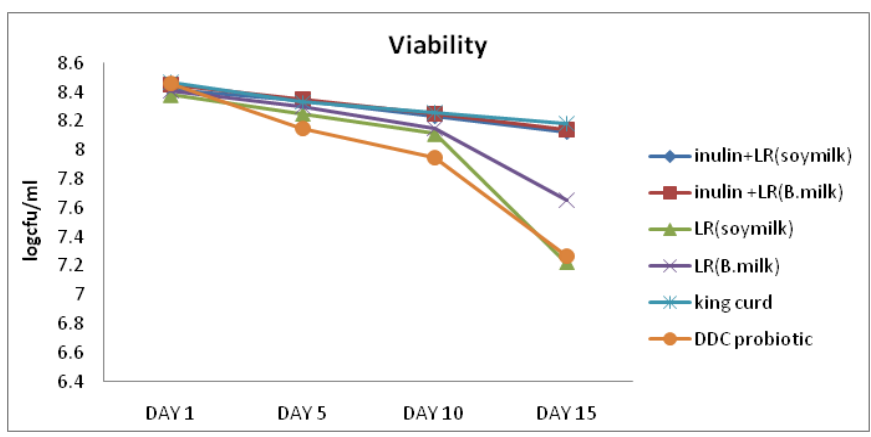

Figure 8. Viability of Probiotics in different products

> Inulin+LR (soymilk) represents all soymilk based synbiotic products

$>$ Inulin+LR (B.milk) represents all buffalo milk based synbiotic products

$>$ LR (soymilk) represents all probiotic soy yoghurts

$>$ LR (B.milk) represents all probiotic buffalo yoghurts

$>$ King curd represents Juju dhau

$>$ DDC probiotic represents DDC probiotic yoghurts

\section{Anti-radical Activity}

Figure 9 depicts that the concentration of BHT showed $50 \%$ inhibition of DPPH which is $0.1 \mathrm{mM}$. While comparing the value obtained from the samples with BHT, the inhibition shown by soymilk based synbiotic products was at the range of 41-45\%. Similarly, the inhibition shown by the buffalo milk based synbiotic products, probiotic buffalo yoghurts, Juju dhau and probiotic yoghurt (DDC) was within the range of 9.5-12\%. Among all the samples the highest inhibition was shown by probiotic soy yoghurts at the range of 68-70\% and soy yoghurts containing L.rhamnosus as probiotic (Table 1).

So, we can conclude that the antioxidant activity was higher in soy based products than in buffalo milk based products. Soybean or soy based products contain high amount of antioxidants than buffalo milk based products due to the presence of isoflavones (Daidzein, genistein) in soy products which acts as antioxidants and are responsible for antioxidant activity.BHT was used as standard antioxidant and solutions with different concentrations of BHT was prepared in order to compare the absorbance of the BHT solutions with the samples and hence on the basis of the relation between absorbance and different concentrations of the BHT solutions, the amount of the antioxidants in the samples was determined (Table 10).

Table 1 shows the effect of percentage inhibition in various yoghurt products prepared by using probiotics strains (L.rhamnosus, A1B and $\mathrm{MgA}$ ) in combination with prebiotics either by using soymilk or buffalo milk as a substrate. The addition of prebiotics (lactulose, sunfibre, and inulin) in soymilk irrespective of the probiotics used in the study did not show significant difference (40.43 $44.81 \%$ ) inhibition. Similarly, the synbiotic yoghurt prepared by using buffalo milk and various prebiotics and various probiotic strains as explained above was found between $(9.84-11.57 \%)$ inhibitions.

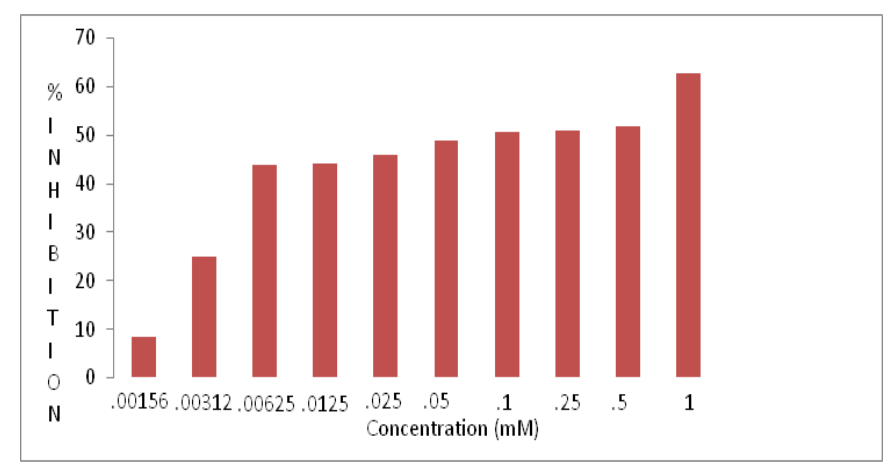

Figure 9. Relation between $\%$ inhibition and concentrations of BHT 
Karki et al.: J. Food Sci. Technol. Nepal, Vol. 8 (23-29), 2013

Table1. Percentage Inhibition of Antioxidant against DPPH

\begin{tabular}{|c|c|c|c|}
\hline Sample & Prebiotic & Probiotic & $\%$ inhibition \\
\hline & \multirow{3}{*}{ Lactulose } & L.rhamnosus & 44.41 \\
\hline & & A1B strain & 42.82 \\
\hline & & $\mathrm{MgA}$ strain & 43.08 \\
\hline \multirow{9}{*}{ Soymilk } & \multirow{3}{*}{ Sunfibre } & L.rhamnosus & 44.81 \\
\hline & & A1B strain & 41.62 \\
\hline & & MgA strain & 40.43 \\
\hline & \multirow{4}{*}{ Inulin } & L.rhamnosus & 41.22 \\
\hline & & A1B strain & 43.22 \\
\hline & & MgA strain & 43.75 \\
\hline & & L.rhamnosus & 10.10 \\
\hline & Lactulose & A1B strain & 9.84 \\
\hline & & MgA strain & 10.37 \\
\hline \multirow[t]{6}{*}{ Buffalo milk } & \multirow{3}{*}{ Sunfibre } & L.rhamnosus & 9.84 \\
\hline & & A1B strain & 10.77 \\
\hline & & MgA strain & 10.10 \\
\hline & \multirow[t]{3}{*}{ Inulin } & L.rhamnosus & 11.70 \\
\hline & & A1B strain & 11.17 \\
\hline & & MgA strain & 11.57 \\
\hline \multirow{4}{*}{ Soymilk } & & L.rhamnosus & 69.14 \\
\hline & & A1B strain & 68.75 \\
\hline & & MgA strain & 68.08 \\
\hline & & L.rhamnosus & 9.70 \\
\hline \multirow[t]{2}{*}{ Buffalo milk } & & A1B strain & 9.97 \\
\hline & & MgA strain & 9.84 \\
\hline Juju dhau & & & 10.23 \\
\hline Probiotic (DDC) & & & 9.84 \\
\hline
\end{tabular}

\section{Conclusion}

Based on the comparison of the characteristics of all yoghurts along with their antiradical activity, it was found that synbiotic soy youghurt with L.rhamnosus as probiotic and inulin as prebiotic was the most suitable product in all respect. Hence, probiotic and prebiotic can be used in appropriate combination to make a synbiotic product with health benefits due to the presence of high concentration of isoflavones which was evident from higher anti-radical activity. Moreover, the stability of our synbiotic soy yoghurt was higher than the probiotic yoghurt available in the local market of Nepal.. Also, synbiotic yoghurt prepared from buffalo milk can be as good as Juju dhau.

\section{References}

Aggawala A.C. and Sharma R.M. (1961). A Laboratory Manual of Milk Inspection. Bombay, Calcutta, New Delhi, India

AOAC (2005). Official methods of analysis. The association of official analytical chemists. 16 th edition. 481. North Fredrick Avenue Gaithersburg, Maryland, USA

APHA (1960). Standard Methods for the Examination of Water and Waste Water (A. E. Eaton, L. S. Clesceri and A.E. Greenberg, eds.). American public health association, Maryland, United Book Press Inc 
Chow J. (2002). Probiotics and prebiotics: A brief overview. J Ren Nutr 12, 76-86

Daniel J.R. (2010). Physical Properties of Foods. Department of Foods and Nutrition. Purdue University

Hsieh H.M., Wu W.M. and Hu M.L. (2009). Soy isoflavones attenuate oxidative stress and improve parameters related to aging and Alzheimer's disease in C57BL/6J mice treated with D-galactose (vol 47, pg 625, 2008). Food and Chemical Toxicology 47, 2686-2687.

Kanthamani S., Nelson A. I. and Steinberg M. P. (1978). Home preparation of soymilk: a new concept. Whole soybean foods for home and village use. Intsoy. 14 (5): 5-9

Kwok K. and Niranjan K. (1995). Review: Effect of thermal processing on soymilk. International J Food Sci. and Technol. 30: 263

Liu K. (1997). Soybeans: Chemistry, Technology and Utilization. Chapman and Hall, New York

Molyneux P. (2004). The use of stable free radical diphenylpicrylhydrazyl (DPPH) for estimating antioxidant activity. Songklanakarin Journal of Science Technology, (26 (2): 211-219

Pyo Y. H. and Song S.M. (2009). Physicochemical and Sensory Characteristics of a Medicinal Soy Yogurt Containing Health-Benefit Ingredients, J. Agric. Food Chem. 57 (1), 170-175

Rosenthal, Amauri; Deliza, Rosires; Cabral, Lourdes M. C.; Cabral, Lair C.; Farias, Carlos A. A.; Domingues, Aline M. (2003). Effect of enzymatic treatment and filtration on sensory characteristics and physical stability of soymilk. Food Control. 14(3): 187

Trindade C.S.F., Terzi S.C., Trugo L.C., Della Modesta R.C. and Couri S. (2001). Development and sensory evaluation of soy milk based yoghurt. Arch Latinoam Nutr 51, 100-104.

Verma S. and Palanchoke S. (2007). Synbiotics potential dietary supplements in functional foods, $1-7$ 